\title{
Efeitos da vitimização por pares sobre o uso de substâncias psicoativas e comportamentos violentos em adolescentes
}

\author{
Cristina Lessa Horta. Pontifícia Universidade Católica do Rio Grande do Sul \\ Rogério Lessa Horta. Universidade FEEVALE \\ Daniela Centenaro Levandowski. Universidade Federal de Ciências da Saúde de Porto Alegre \\ Vanessa Andina Teixeira. Centro Universitário Ritter dos Reis \\ Carolina Saraiva de Macedo Lisboa. Pontifícia Universidade Católica do Rio Grande do Sul
}

\section{Resumo}

Estudo transversal, de base escolar, que investigou se vitimização por pares associa-se à policonsumo de substâncias e envolvimento em comportamentos violentos (brigas e/ou porte de armas). Participaram 3.547 escolares do interior do Rio Grande do Sul, com idade média de 14 anos $(d p=1,66)$. Realizou-se amostragem aleatória, estratificada por sexo, idade, município e rede de ensino. Os dados foram coletados em 2012, através de questionário autoaplicável. Foram realizadas análises descritivas, através do teste Chi Quadrado de Pearson, e modelo de equações estruturais. Evidenciou-se que vitimização associa-se ao policonsumo apenas quando há sofrimento psíquico e relação negativa com pai e/ou mãe, associando-se ao maior envolvimento com comportamentos violentos tanto de forma direta como mediada pela relação com os pais e pela saúde mental dos escolares. Conclui-se que a vitimização parece instigar comportamentos violentos, denunciando a existência de um ciclo vicioso de agressividade, o que reforça a importância do seu enfrentamento.

Palavras-chave: bullying; drogas; relações familiares; violência; adolescência.

\begin{abstract}
Effects of peer vitimization on the use of psychoactive substances and violent behavior in adolescents. A cross-sectional, schoolbased study that evaluated whether victimization by peers is associated with polydrug use and involvement in violent behavior (fights and/or carrying arms). A total of 3,547 students from the interior of Rio Grande do Sul, with a mean age of 14 years $(S D=1.66)$ participated. Sampling was randomized, stratified by gender, age, municipality and educational network. Data were collected in 2012, through a self-administered questionnaire. Descriptive analyzes were performed using the Pearson's Chi Square test and a Structural Equations Model. It has been shown that victimization is associated with polydrug use only when there is psychological distress and negative relationship with father and/or mother, and is associated with the greater involvement with violent behaviors even directly or mediated by the adolescents' relationship with parents and adolescents' mental health. It is concluded that victimization seems to foster violent behavior, denouncing the existence of a vicious cycle of aggression and reinforcing the importance of being confronted.
\end{abstract}

Keywords: bullying; drugs; family relations; violence; adolescence.

\section{Resumem}

Efectos de la vitimización por pares sobre el uso de sustancias psicoativas y comportamientos violentos en adolescentes. Estudio transversal, de base escolar, que investigó si la victimización por pares se asocia al policonsumo de sustancias y la participación en comportamientos violentos (peleas y/o porte de armas). Participaron 3.547 escolares del interior de Rio Grande do Sul, con una edad promedio de 14 años $(d p=1,66)$. Se realizó muestreo aleatorio, estratificado por sexo, edad, municipio y red de enseñanza. Los datos fueron recogidos en 2012 a través de cuestionario autoadministrado. Se realizaron análisis descriptivos, a través del test Chi cuadrado de Pearson, y el modelo de ecuaciones estructurales. Se evidenció que la victimización se asocia al policonsumo sólo cuando hay sufrimiento psíquico y relación negativa con padre y / o madre, asociándose al mayor involucramiento con comportamientos violentos tanto de forma directa como mediada por la relación de los adolescents con los padres y la salud mental de los adolescentes. Se concluye que la victimización parece instigar los comportamientos violentos, denunciando la existencia de un ciclo vicioso de agresividad, lo que reforza la importancia de su enfrentamiento.

Palabras clave: bullying; drogas; relaciones familiares; violencia; adolescentes. 
Durante a adolescência, sentir-se aceito pelos amigos e afastar-se saudavelmente dos pais ou cuidadores é fundamental para a autoestima e o processo de aquisição da identidade e da autonomia. Já a exclusão social associa-se a sintomas de sofrimento psíquico, tais como depressão (Forlim, Stelko-Pereira, \& Williams, 2014), ansiedade (Crawford \& Manassis, 2011) e comportamento antissocial (Cruzeiro et al., 2008), acarretando prejuízos emocionais e sociais para o desenvolvimento do adolescente (Berger, Lisboa, Cuadros, \& Tezanos-Pinto, 2016). Nessa direção, receber apelidos pejorativos, ser ameaçado, roubado ou ter seus pertences destruídos, ser excluído de jogos ou grupos, ser agredido fisicamente, ser provocado, ser alvo de fofoca e ser forçado por alguém a fazer algo contra a própria vontade são situações que caracterizam a vitimização por pares e podem levar à exclusão social do adolescente.

Fazer uso de substâncias psicoativas é uma das formas que frequentemente os jovens encontram para aliviar sentimentos indesejados, como aqueles decorrentes do desamparo e da rejeição do grupo de pares (Hong \& Espelage, 2012). Há estudos evidenciando que sofrer vitimização por pares aumenta a possibilidade de ocorrência de uso de substâncias. No entanto, há também estudos indicando ausência de associação entre esses fenômenos e ainda outros indicando que vítimas fazem menos uso de substâncias, quando comparadas a não vítimas ou a indivíduos não envolvidos com situações de violência entre pares (C. L. Horta et al., 2018). A divergência entre os resultados dos estudos indica a existência de uma relação dinâmica entre esses fenômenos, cujos fatores de interferência precisam ser conhecidos.

Ainda que o uso de substâncias seja percebido, em todas as idades, como recreativo e não relacionado a qualquer problema, entre jovens brasileiros este comportamento é mais referido quando há sentimentos de solidão e ausência de amigos próximos (Malta et al., 2014). Trata-se, portanto, de um comportamento também relacionado à influência grupal. Embora integrar um grupo que usa ou abusa de substâncias não seja necessário para o próprio uso, a convivência ou pressão grupal pode elevar esse risco, tanto pela simples oferta que é feita pelos parceiros, como pela função integradora desse uso (Malta et al., 2014; Vázquez Fernández et al., 2014).

Durante a adolescência, o início do uso de substâncias também costuma ocorrer pela necessidade de transgredir ou mesmo por curiosidade (Vázquez Fernández et al., 2014). No Brasil, o primeiro contato com álcool e tabaco tem ocorrido aos 13 anos de idade, sendo que, entre os 14 e 15 anos, já são referidos contatos com maconha e cocaína (Carlini et al., 2010). A precocidade desse contato expõe o jovem a um maior risco de desenvolver problemas de saúde e eleva o risco de consumo excessivo ao longo da vida, pois o uso tende a aumentar conforme a idade (Malta et al., 2014), levando ao policonsumo. O policonsumismo, definido como o uso de mais de um tipo de substância psicoativa, simultaneamente ou não (Vázquez Fernández et al., 2014), já foi constatado como padrão de uso entre escolares da Espanha (Vázquez Fernández et al., 2014) e preocupa os profissionais da saúde. Além de dificultar a intervenção terapêutica, costuma acarretar o uso de substâncias mais prejudiciais à saúde e envolver maior risco de desenvolvimento de transtornos associados ao uso, tais como sofrimento psíquico, depressão, ansiedade e condutas violentas (Cruzeiro et al., 2008; Vázquez Fernández et al., 2014).

Outro fenômeno referido em estudos, e que preocupa organizações envolvidas na promoção da saúde dos jovens, é o envolvimento em situações de perpetração de violência (Ministério da Saúde [MS], 2010). Dados indicam uma correlação positiva entre praticar atos de violência física e início precoce do uso de álcool, uso abusivo de álcool, ter amigos com condutas antissociais, recente vitimização por pares e envolvimento em situações perigosas em busca da sensação de prazer (Herrenkohl, Hemphill, Mason, Toumbourou, \& Catalano, 2012). A perpetração da violência física também parece associada a relatos de conflitos familiares e pouco ou nenhum monitoramento parental em relação aos comportamentos do adolescente (Herrenkohl et al., 2012). Por outro lado, viver com pai e mãe parece proteger os adolescentes de sofrer agressões físicas (Romaní \& Gutiérrez, 2010), assim como coabitar com pelo menos um dos genitores mostra-se protetivo contra o porte de armas (R. L. Horta, Horta, Pinheiro, \& Krindges, 2010). Embora o comportamento isolado de portar armas não indique um ato violento, evidencia a predisposição para o envolvimento em situações dessa natureza, além de indicar um monitoramento familiar precário, que possibilita o acesso a armas e não impede os jovens de carregá-las consigo (R. L. Horta et al., 2010).

Sofrimento psíquico e dificuldades na relação com os pais (ou cuidadores) estão, portanto, associados ao uso de substâncias e ao envolvimento em comportamentos 
violentos durante a adolescência, seja como vítima ou agressor. O tipo de vínculo estabelecido entre pais e filhos desde os primeiros anos de vida tem se mostrado um importante fator para a promoção da saúde mental em todas as faixas etárias (Jordão \& Ramires, 2010). Neste sentido, fazer as refeições com os pais, residir com os mesmos e estes saberem onde os filhos estão no seu tempo livre tende a proteger escolares brasileiros em relação ao uso de substâncias psicoativas (Malta et al., 2014). Da mesma forma, a satisfação dos filhos em estar com a família mostra-se negativamente correlacionada a xingar os colegas, participar de brigas, presenciar brigas na escola, fazer uso de substâncias, sofrer ameaças na escola e sentir prazer em brigar (Tortorelli, Carreiro, \& Araújo, 2010). O apoio parental também protegeu escolares americanos de sofrerem bullying (Wang, lannotti, \& Nansel, 2009).

Por outro lado, uma relação com os pais pautada pela insegurança deixa adolescentes em situação de vulnerabilidade emocional e afetiva (Jordão \& Ramires, 2010), o que pode predispor ao abuso de substâncias e ao envolvimento em comportamentos violentos, especialmente entre aqueles que são vitimizados. Modelos familiares caracterizados por autoritarismo, violência, conflitos familiares, comunicação negativa entre os membros, falta de contenção e orientação parecem positivamente associados ao envolvimento de adolescentes em situações de violência na escola e uso de drogas (Benchaya et al., 2011; Potocnjak, Berger, \& Tomicic, 2011).

Embora a literatura indique associação entre vitimização por pares, uso de substâncias e envolvimento em comportamentos violentos, divergências observadas entre os estudos permitem supor que outros fatores, tais como a qualidade da relação com os pais e a saúde mental dos jovens, interferem nessa associação. A possibilidade de articular estas variáveis em um modelo de análise levou à proposição deste estudo, que pretendeu verificar se a percepção de uma relação negativa com os pais e a presença de sofrimento psíquico aumentam o envolvimento de adolescentes escolares, que sofreram vitimização por pares, com o uso de substâncias psicoativas ou comportamentos violentos (especificamente, envolvimento em brigas e porte e/ou uso de armas). Ao elucidar a relação dinâmica existente entre esses fenômenos, busca-se oferecer ferramentas para a elaboração de intervenções psicológicas de cunho preventivo que visem o enfrentamento dessas situações de risco.

\section{Método}

Este artigo analisa dados oriundos de um estudo maior, com delineamento transversal de base escolar, realizado no ano de 2012, no qual foram entrevistados estudantes das escolas dos municípios de Lajeado e Sapiranga, ambos de médio porte, localizados no interior do Estado do Rio Grande do Sul (C. L. Horta et al., 2015). O banco de dados original foi constituído por amostragem aleatória por turma, preservando-se a proporcionalidade da amostra por sexo, idade, município e rede de ensino (pública municipal, pública estadual e privada). O cálculo amostral original previa estimativas de prevalência de uso de substâncias na vida variando de 1,5\% para o crack até $65 \%$ para bebidas alcoólicas, com erro aceitável variando de $0,5 \%$ para o crack a $2 \%$ para álcool, com nível de confiança de $95 \%$ e poder de $80 \%$. Foram entrevistados 3547 escolares, com idades entre 12 e 17 anos, já descontados 9,8\% de perdas por preenchimento insuficiente ou reprovação nos procedimentos de controle de qualidade.

O questionário aplicado aos adolescentes em sala de aula foi desenvolvido com base em instrumentos utilizados em grandes levantamentos nacionais, padronizado e pré-testado, com foco no uso no ano de substâncias psicoativas pelos escolares e condições associadas a este fenômeno. Os instrumentos foram digitados no programa Epidata 3.5 com dupla entrada, para checagem e correção de eventuais erros de digitação.

Todos os cuidados éticos foram tomados a fim de garantir a liberdade para participar ou não no estudo, assim como o anonimato e o bem-estar dos escolares. A coleta de dados em cada município gerou projetos distintos, aprovados pelo Comitê de Ética em Pesquisa da Universidade do Vale do Rio dos Sinos (UNISINOS), sob os protocolos 074/2011 e 028/2012. Este recorte analítico foi aprovado pelo Comitê de Ética em Pesquisa da Pontifícia Universidade Católica do Rio Grande do Sul (PUCRS), sob o protocolo 1.391.831.

O modelo teórico proposto para o presente estudo verificou a relação entre as variáveis a seguir apresentadas. Foram consideradas variáveis endógenas aquelas passíveis de serem explicadas por uma ou mais variáveis exógenas. Já as variáveis exógenas estão ligadas ao desfecho estudado, porém não sofrem influência de outras variáveis incluídas no modelo. 


\section{Variável Endógena de Exposição}

Vitimização por pares - variável dicotômica, com respostas do tipo SIM ou NÃO para a pergunta "Alguma vez na vida você já foi hostilizado, agredido ou maltratado por colegas da escola?".

\section{Variáveis Endógenas de Desfechos}

a) Número de comportamentos violentos: variável criada a partir da soma das respostas às questões "No último ano, você esteve envolvido em algum tipo de briga?" e "No último ano, você usou ou carregou com você algum tipo de arma (canivete, faca, revólver, pistola ou qualquer outro)?". Ambas as questões eram dicotômicas, com respostas tipo SIM ou NÃO, onde NÃO = 0 e SIM = 1. Na variável obtida, estabeleceu-se que: 0 = não ter referido nenhum dos comportamentos estudados; 1 = ter referido envolvimento em briga ou uso de arma no último ano; 2 = ter referido briga e uso de arma no último ano.

b) Número de substâncias usadas no ano: criada a partir das respostas do tipo SIM ou NÃO às questões relacionadas a ter feito uso de alguma das seguintes substâncias no último ano: tabaco, álcool, maconha, cocaína, solventes ou ecstasy. Para a análise descritiva, a variável foi categorizada em nenhuma, uma e duas ou mais (policonsumo) das substâncias estudadas. Para a inserção no modelo de equações estruturais, a variável foi codificada com escores que variam de 0 a 6 , sendo 0 a resposta "não" ao uso de qualquer uma das drogas investigadas e 6 a resposta "sim" ao uso de todas as drogas no último ano.

\section{Variáveis Endógenas Mediadoras}

a) Relação com pai e mãe: variável criada a partir das respostas às questões: "Como é o seu relacionamento com o seu pai?" e "Como é o seu relacionamento com a sua mãe?". Para ambas as questões, os escolares poderiam assinalar uma das seguintes opções de resposta: "Não tenho contato com meu pai/minha mãe" ou "Quando estou com meu pai/minha mãe é: ( ) ótimo ( ) bom ( ) regular ( ) ruim ( ) péssimo". Dos 3547 questionários analisados, 82 indicaram não ter contato com pai e/ou mãe, sendo considerados missing no emprego desta variável, que foi categorizada da seguinte forma: positiva com ambos (quando os esco- lares responderam ótimo, bom ou regular nas duas questões), negativa com mãe ou com pai (quando responderam ruim ou péssimo apenas para uma das questões) e negativa com ambos (quando responderam ruim ou péssimo nas duas questões).

b) Sofrimento psíquico (escore no SRQ-20): avaliado a partir das respostas às 20 questões do Self-Reporting Questionnaire (SRQ-20) (Mari \& Williams, 1986), todas categóricas e dicotômicas, com respostas tipo SIM ou NÃO, sobre sintomas emocionais e físicos associados a transtornos psiquiátricos. O SRQ-20 é utilizado para o rastreamento de transtorno mental não-psicótico; quanto maior a pontuação na soma dos 20 itens, maior a possibilidade de apresentação de alguma forma de adoecimento (Santos, Araújo, Pinho, \& Silva, 2010). $\mathrm{Na}$ análise descritiva, a variável foi denominada "sofrimento psíquico" por ter sido categorizada de acordo com o ponto de corte (escores iguais ou inferiores a 6 foram considerados indicativos de ausência de sofrimento psíquico, enquanto escores iguais ou superiores a 7 pontos indicaram presença de sofrimento psíquico). No modelo de equações estruturais, a variável foi denominada "escore no SRQ-20", por ter sido apresentada de forma contínua, codificada com escores que variam de 0 a 20, sendo 0 a resposta indicativa de ausência de sofrimento psíquico e 20 a resposta indicativa de sofrimento psíquico máximo. Convém salientar que o coeficiente alfa de Cronbach do SRQ-20 foi de 0,81.

\section{Variáveis Exógenas}

Incluídas para fins de controle de fatores de confusão:

a) Idade: variável numérica discreta, considerada em anos completos (12 a 17 anos).

b) Sexo: variável nominal binária, considerando-seo sexo autoreferido como masculino ou feminino.

Os dados foram analisados através do programa Stata versão 13.0, utilizando-se, inicialmente, análise descritiva, para verificar a distribuição da amostra segundo as variáveis em estudo. Foi também analisado o comportamento dos desfechos "número de substâncias usadas no ano" e "número de comportamentos violentos praticados no ano" segundo as variáveis independentes e a exposição de interesse. Conforme descrito anteriormente, nesta etapa a variável foi categorizada em nenhuma, uma e duas 
ou mais das substâncias estudadas (álcool, tabaco, maconha, cocaína, solventes ou ecstasy).

Em seguida, as variáveis integraram um modelo de equações estruturais (Figura 1), tendo a vitimização por pares como exposição. Este modelo de análise foi proposto a fim de identificar a relação entre as variáveis estudadas, pois permite examinar múltiplas relações simultaneamente através de análise fatorial confirmatória (CFA) e análise de regressão (Acock, 2013). Os desfechos foram incluídos no modelo na seguinte ordem: número de comportamentos violentos praticados no ano e número de substâncias usadas no ano. A inclusão do segundo desfecho mostrou associação entre as variáveis, mas os índices indicativos de ajuste do modelo não foram adequados. Realizou-se, então, uma covariância entre número de comportamentos violentos e número de substâncias usadas no ano, por sugestão do programa, estando de acordo com a literatura revisada (C. L. Horta et al., 2015). Novamente o modelo mostrou indicadores de ajuste adequado.

Em seguida, foram incluídas no modelo as variáveis "escore no SRQ-20" como variável endógena, por sofrer a influência da vitimização por pares e influenciar os desfechos estudados (Crawford \& Manassis, 2011; Forlim et al., 2014). Do mesmo modo, adicionou-se a variável "relação com pai e mãe" como variável endógena, pois há indicativo de que esta pode potencializar ou minimizar o efeito da vitimização sobre o uso de substâncias (Romaní \& Gutiérrez, 2010; Wang et al., 2009). A associação entre vitimização por pares e avaliação da relação com pai e/ou mãe foi, ainda, confirmada através do teste de Chi-quadrado de Pearson $\left[x^{2}(2)=36,648, p=0,00\right.$, que indicou que os escolares que referiram situação de vitimização por pares na vida avaliaram pior a relação com pai e/ou mãe, reforçando a presença desta variável no modelo.

Novamente foi realizada uma covariância, desta vez entre "escore no SRQ-20" e "relação com pai e mãe". O modelo mostrou-se bem ajustado e foram adicionadas as variáveis exógenas idade e sexo como controles para fatores de confusão, uma de cada vez e nesta ordem. Os índices referentes ao ajuste do modelo final foram: RMSEA - Root mean squared error of approximation <0,001; CFI - Comparative fit index =1,000; SRMR - Standardized root mean squared residual $<0,001$ (Kline, 2005).

\section{Resultados}

A análise descritiva evidenciou que 50,7\% $(n=1799)$ dos escolares disseram não ter feito uso de substâncias psicoativas no último ano. Entre os $49,3 \%$ ( $n=1748$ ) que fizeram uso de substâncias, a maioria utilizou apenas um tipo de droga (36,4\%, $n=1291$ ), sendo o policonsumo referido por $12,9 \%(n=457)$. O policonsumo caracterizou-se da seguinte forma: $7,6 \%(n=269)$ dos escolares usaram dois dos seis tipos de substâncias investigadas, 3,1\% ( $n=112)$ usaram três tipos, $1,5 \%(n=53)$ fizeram uso de quatro tipos, $0,5 \%(n=17)$ usaram cinco tipos e $0,2 \%(n=6)$ usaram todos os seis tipos de substâncias no último ano.

Quanto ao número de comportamentos violentos no ano, $73,9 \%(n=2577)$ da amostra referiu não ter se envolvido em brigas nem ter portado armas. Dos $26,1 \%(n=911)$ que se envolveram em comportamentos violentos, $20,5 \%(n=716)$ referiram apenas um dos comportamentos estudados, enquanto $5,6 \%(n=195)$ referiram brigas e porte de armas.

A Tabela 1 evidencia que todas as variáveis selecionadas a partir da revisão teórica para inclusão no modelo de equações estruturais estão associadas com os desfechos policonsumo de substâncias e comportamentos violentos no último ano. Entretanto, embora a diferença entre meninos e meninas tenha sido estatisticamente significativa, observou-se um padrão semelhante para os dois grupos, com predomínio de relatos de ausência de experimentação de substâncias. Ainda que o policonsumo seja menor que o uso de apenas um tipo de droga em todas as idades, os percentuais referentes a este comportamento aumentam à medida que a idade avança. Da mesma forma, aumenta o envolvimento com os dois comportamentos violentos estudados à medida que a idade avança, diminuindo o envolvimento com apenas um destes comportamentos. Os dados apresentados na Tabela 1 também evidenciam uma tendência pouco expressiva das meninas de fazerem mais uso de substâncias no ano, quando comparadas aos meninos. No entanto, os meninos referiram maior envolvimento com os comportamentos violentos no ano, quando comparados às meninas. 
Tabela 1. Distribuição dos Escolares de 12 a 17 Anos de Idade Segundo as Variáveis Independentes e sua Relação com o Número de Substâncias Usadas no Ano e o Número de Comportamentos Violentos Praticados no Ano, 2012 (N=3547)

\begin{tabular}{|c|c|c|c|c|c|c|c|c|}
\hline \multirow{2}{*}{ Variáveis } & \multicolumn{2}{|c|}{ Distribuição } & \multicolumn{3}{|c|}{ Número de substâncias usadas } & \multicolumn{3}{|c|}{ Número de comportamentos violentos } \\
\hline & $\mathbf{N}$ & $\%$ & $n(\%)$ & $n(\%)$ & $n(\%)$ & $\mathrm{n}(\%)$ & $n(\%)$ & $n(\%)$ \\
\hline Idade em anos & & & & & $\mathrm{p}<0,001^{*}$ & & & $\mathrm{p}=0,001^{*}$ \\
\hline 12 & 937 & $26,4 \%$ & $723(77,2 \%)$ & $167(17,8 \%)$ & $47(5,0 \%)$ & $716(76 \%)$ & $196(21,3 \%)$ & $25(2,7 \%)$ \\
\hline 13 & 611 & $17,2 \%$ & $388(63,5 \%)$ & $168(27,5 \%)$ & $55(9,0 \%)$ & $440(71,5 \%)$ & $135(22,5 \%)$ & $36(6,0 \%)$ \\
\hline 15 & 541 & $15,3 \%$ & $204(37,7 \%)$ & $244(45,1 \%)$ & $93(17,2 \%)$ & $396(73 \%)$ & $107(19,9 \%)$ & $38(7,1 \%)$ \\
\hline 16 & 508 & $14,3 \%$ & $119(23,5 \%)$ & $279(54,9 \%)$ & $110(21,6 \%)$ & $383(74,8 \%)$ & $96(19,3 \%)$ & $29(5,9 \%)$ \\
\hline 17 & 309 & $8,7 \%$ & $74(23,9 \%)$ & $164(53,1 \%)$ & $71(23,0 \%)$ & $241(77,9 \%)$ & $47(15,3 \%)$ & $21(6,8 \%)$ \\
\hline Sexo & & & & & $p=0,035^{*}$ & & & $p<0,001^{*}$ \\
\hline Até 6 pontos & 2073 & $60,8 \%$ & $1199(57,9 \%)$ & $695(33,5 \%)$ & $179(8,6 \%)$ & $1628(78,3 \%)$ & $356(17,4 \%)$ & $89(4,3 \%)$ \\
\hline 7 ou mais pontos & 1335 & $39,2 \%$ & $513(38,5 \%)$ & $561(42,0 \%)$ & $261(19,5 \%)$ & $916(68,3 \%)$ & $324(24,5 \%)$ & $95(7,2 \%)$ \\
\hline Relação com pais & & & & & $\mathrm{p}<0,001^{*}$ & & & $p<0,001^{*}$ \\
\hline Positiva com ambos & 2603 & $75,1 \%$ & $1404(54 \%)$ & $935(35,9 \%)$ & $264(10,1 \%)$ & 2025 (77.5\%) & $475(18,5 \%)$ & $103(4,0 \%)$ \\
\hline Negativa com um & 727 & $21,0 \%$ & $311(42,8 \%)$ & $274(37,7 \%)$ & $142(19,5 \%)$ & $487(66,4 \%)$ & $172(24,1 \%)$ & $68(9,5 \%)$ \\
\hline Negativa com ambos & 135 & $3,9 \%$ & $38(28,2 \%)$ & $61(45,1 \%)$ & $36(26,7 \%)$ & $68(50,4 \%)$ & $50(37,0 \%)$ & $17(12,6 \%)$ \\
\hline Vitimização & & & & & $\mathrm{p}=0,004^{*}$ & & & $p<0,001^{*}$ \\
\hline Não & 2639 & $75,3 \%$ & $1377(52,2 \%)$ & $933(35,3 \%)$ & $329(12,5 \%)$ & $2040(77,2 \%)$ & $473(18,0 \%)$ & $126(4,8 \%)$ \\
\hline
\end{tabular}

Em relação à presença de indicadores de sofrimento psíquico, 39,2\% $(n=1.335)$ dos escolares apresentaram escores compatíveis com esta situação, a qual se mostrou associada ao envolvimento em policonsumo de substâncias e comportamentos violentos. Quanto à vitimização por pares no ano, $24,7 \%(n=868)$ dos escolares referiram ter passado por esta situação. Escolares vitimizados fizeram mais uso de substâncias e se envolveram em mais comportamentos violentos do que aqueles que não referiram vitimização por pares no ano.

Quanto à relação com pai e mãe, a maioria dos escolares $(75,1 \%, n=2.603)$ referiu relação positiva com ambos, enquanto apenas $3,9 \%(n=135)$ consideraram negativa a relação com ambos. Nota-se que a ocorrência de policonsumo de substâncias e comportamentos violentos aumenta à medida que piora a relação com os pais, sendo menor entre aqueles que referiram boa relação com ambos, seguido pelos que referiram relação negativa com apenas um.

O modelo de equações estruturais testado (Figura 1) evidenciou associação entre os fenômenos estudados. Destaca-se que não há efeito direto da vitimização por pares sobre o número de substâncias psicoativas usadas no ano. Praticamente $100 \%$ do efeito total da vitimização sobre o número de substâncias usadas é efeito indireto, ou seja, é mediado pela relação negativa com pai e/ou mãe e pela presença de sofrimento psíquico. No entanto, $60,3 \%$ do efeito total da vitimização sobre o número de comportamentos violentos praticados (brigas e/ou uso de armas) é efeito direto (Tabela 2). 


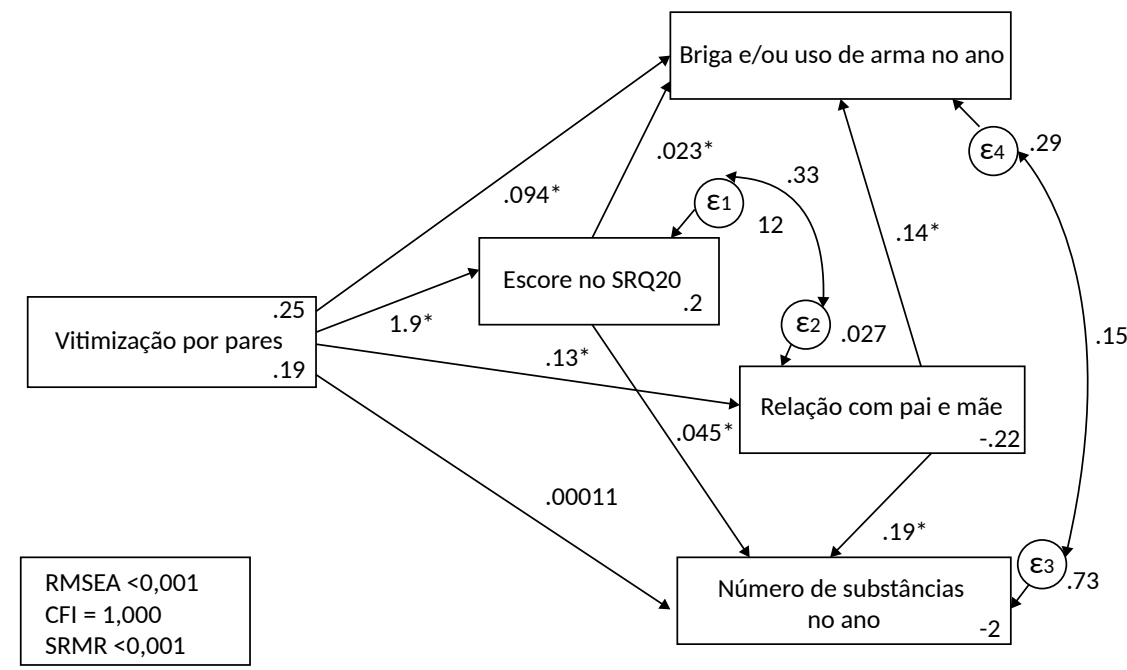

Figura 1. Modelo de Equações Estruturais.

${ }^{*} \mathrm{p}<0,001$

** Modelo ajustado para Idade e Sexo

Tabela 2. Efeitos Estimados para Vitimização por Pares (VPP) sobre Número de Substâncias no Ano (NSA) e Briga e/ou Uso de Arma no Ano (BAA) Entre Escolares de 12 a 17 Anos, 2012 ( $N=3547)$

\begin{tabular}{lccc}
\hline \multicolumn{1}{c}{ Desfechos } & Efeito Direto & $\begin{array}{c}\text { Efeito } \\
\text { Indireto* }\end{array}$ & Efeito Total \\
\hline $\begin{array}{l}\text { Número de Substan- } \\
\text { cias no ano (NSA) }\end{array}$ & $-0,0001$ & $0,111^{* *}$ & $0,111^{* * *}$ \\
$\begin{array}{l}\text { VPP } \rightarrow \text { NSA } \\
\text { Briga ou Uso de Arma } \\
\text { no Ano (BAA) }\end{array}$ & $0,094^{* *}$ & $0,061^{* *}$ & $0,156^{* *}$ \\
$\begin{array}{l}\text { VPP } \rightarrow \text { BAA } \\
{ }^{*} \text { Efeito Indireto via Escore no SRQ20 e Relação com pai e mãe; }{ }^{* *} p<0,001 ;{ }^{* * *} p<0,005 .\end{array}$
\end{tabular}

\section{Discussão}

O principal resultado deste estudo indica que a vitimização por pares tem efeito direto e indireto sobre os comportamentos violentos e predomínio de efeito indireto sobre o policonsumo de substâncias. Este dado contribui para os esforços de diminuição do envolvimento de jovens em tais situações, sugerindo maior atenção à ocorrência de vitimização por pares, especialmente em escolas. Diante do reconhecimento de organizações internacionais de que violência e uso de substâncias são problemas de saúde pública (MS, 2010), os achados ganham ainda mais relevância.

$O$ fato de a vitimização ter efeito direto sobre o número de comportamentos violentos sugere que o comportamento violento pode ser assumido pelos escolares como uma resposta a uma violência interpessoal sofrida. Assim, entende-se que combater a violência entre pares pode prevenir também outros comportamentos violentos.

Os resultados encontrados também indicam que pais e mães de escolares que sofreram vitimização por pares devem ser auxiliados, ainda que não possam ser responsabilizados pelo uso de substâncias de seus filhos. Suportar as dificuldades inerentes à adolescência, preservando a capacidade de orientação, apoio e continência em relação às demandas dos filhos, pode auxiliar no enfrentamento das tarefas desenvolvimentais adolescentes (Potocnjak et al., 2011). Igualmente, um nível adequado de saúde mental parece ser capaz de proteger jovens que sofreram vitimização por pares em relação ao policonsumo de substâncias.

Praticamente metade dos escolares (49,3\%; $n=1748)$ disse ter feito uso de alguma substância psicoativa no ano, o que evidencia a alta prevalência do contato com substâncias durante a adolescência, corroborando resultados obtidos em levantamentos brasileiros (Carlini et al., 2010; R. L. Horta et al., 2014). Além da precocidade do envolvimento com o uso, destaca-se o aumento do número de substâncias experimentadas pelos jovens com a idade, mesmo dentro de um perfil etário restrito como o deste estudo. Embora o percentual de policonsumo encontrado neste estudo seja menor que o identificado entre escolares espanhóis (38,3\%; Vásquez Fernandez et al., 2014), é maior que o percentual de $6,9 \%$ encontrado entre adolescentes do Texas (Peinado, Villanos, Singh, \& Leiner 2014). Ainda que apontem diferenças entre as prevalências 
de policonsumo, outros estudos também indicam incremento deste padrão à medida que a idade avança (Malta et al., 2014; Vásquez Fernandez et al., 2014).

Embora não se tenha discriminado o tipo de substância utilizada, considerando-se que o álcool é a substância mais consumida entre os escolares desta amostra (C. L. Horta et al., 2015) e que apenas álcool e tabaco são substâncias consideradas lícitas no Brasil, pode-se inferir que, à medida que a idade avança, aumenta também o contato dos adolescentes com as substâncias ilícitas. Nem todo usuário de duas substâncias consome álcool e tabaco e, a partir de três ou mais substâncias, seguramente uma ou mais são ilícitas.

Entre os escolares deste estudo, as meninas mostraram uma tendência de maior envolvimento que os meninos com o uso no ano de substâncias. Igualmente, Peinado et al. (2014) identificaram que meninas $(77,0 \%)$ do estado do Texas (nos Estados Unidos) fizeram mais uso combinado de álcool e outras drogas, em comparação a meninos (23,0\%; $p=0,015)$. Vásquez Fernandez et al. (2014) também já haviam identificao maior envolvimento das meninas espanholas com o uso de álcool e tabaco, além do policonsumo de duas substâncias. No entanto, naquele estudo, os meninos espanhóis referiram mais uso de substâncias ilícitas e maior envolvimento com o policonsumo de mais de três substâncias.

Ainda que a diferença entre meninas e meninos não seja expressiva, tal resultado não seria esperado em momentos históricos anteriores, nos quais meninos brasileiros apresentavam maior envolvimento que as meninas com o uso de substâncias (Souza \& Silveira Filho, 2007). Esse achado evidencia uma diminuição da repressão cultural em relação às mulheres. Um estudo com universitários portugueses (Brito et al., 2015) apontou a aceitação social do consumo de álcool pelas mulheres e a busca pela igualdade de gênero como motivos para o aumento deste comportamento. Assim, é possível pensar que algumas adolescentes, na construção da identidade feminina e na busca por autonomia e liberdade, façam uso de substâncias (Brito et al., 2015).

Um resultado importante e inédito deste estudo diz respeito à evidência de que a vitimização por pares não está associada diretamente ao policonsumo de substâncias feito pelos escolares. O modelo de equações estruturais testado permitiu verificar que a relação negativa com pai e/ou mãe e a presença de sofrimento psíquico explicam o efeito da vitimização sobre o número de substâncias psicoativas utilizadas no ano. Tais resultados podem justificar a controvérsia identificada entre os estudos que investigaram a associação entre bullying e uso de substâncias, revisados por C. L. Horta et al. (2018). Assim, foi possível verificar que outros fenômenos interferem na relação entre vitimização por pares e número de substâncias usadas. De qualquer modo, entende-se que jovens vitimizados encontram-se em risco para diversas consequências negativas, especialmente na presença de outras condições de vulnerabilidade, o que pode explicar o fato de vítimas fazerem uso de mais substâncias que não vítimas.

Tal resultado é condizente com a literatura disponível. Embora não tenha sido encontrada outra investigação pautada no modelo teórico do presente estudo, a literatura aponta uma boa relação familiar como variável protetora em relação ao uso de substâncias entre adolescentes. Ainda que seja difícil definir uma boa relação com pai e/ou mãe, características como a presença e o interesse dos pais por seus filhos, acompanhamento e supervisão, além de hábitos como fazer pelo menos uma das refeições juntos e clima familiar favorável ao diálogo, protegem adolescentes em relação ao uso de substâncias psicoativas (R. L. Horta et al., 2014; Malta et al., 2014). Por outro lado, Tavares, Béria e Lima (2001) identificaram um relacionamento ruim ou péssimo com pai e mãe como fator de risco para uso de substâncias psicoativas entre os adolescentes por eles estudados. Entretanto, o fato de os autores contarem apenas com a percepção dos jovens sobre a relação com seus pais, pode figurar como uma limitação do presente estudo, pois não se teve acesso à opinião dos pais para uma triangulação dos achados. Além disso, a qualidade da relação não foi avaliada a partir de um instrumento padronizado, que defina claramente o que poderia ser qualificado como bom ou ruim. De todo modo, a percepção dos jovens ao qualificar esta relação parece ser relevante.

A associação encontrada nesse estudo entre sofrimento psíquico e uso de substâncias psicoativas também está em acordo com a literatura científica. Entre os escolares que participaram do levantamento realizado pela PeNSE 2012, insônia e sentimentos de solidão mostraram-se associados ao maior consumo de substâncias (Malta et al., 2014).

Ainda que a maioria dos escolares não tenha se envolvido em brigas ou uso de armas no ano, $26,1 \%$ referiram algum tipo de envolvimento, percentual semelhante ao encontrado entre adolescentes portugueses (Matos, Simões, Camacho, Reis, \& Equipa Aventura Social., 2015) e americanos-mexicanos (Peinado et al., 2014). Em ambos os estudos, os meninos mostraram-se mais envolvidos 
que as meninas com os comportamentos violentos estudados, assim como ocorreu entre os escolares do presente estudo. Tais resultados indicam que, apesar das modificações percebidas na sociedade atual em relação aos papeis de gênero, a violência física ainda parece ser mais utilizada pelos homens como uma forma de resolução de conflitos (Bandeira \& Hutz, 2012; Nascimento, Gomes, $\&$ Rebello, 2009). Tratando-se de adolescentes, é possível que alguns jovens, na construção da identidade masculina, entendam o envolvimento em situações de violência física como uma condição natural para a afirmação da sua masculinidade (Nascimento et al., 2009). Por outro lado, as meninas parecem ainda identificadas com a ideia de que não devem ser violentas (Bandeira \& Hutz, 2012). Cabe destacar que neste estudo não foram investigados comportamentos de violência relacional ou verbal, nos quais as meninas costumam se envolver mais que os meninos (Potocnjak, Berger, \& Tomicic, 2011). Uma vez que as formas de expressão de agressividade feminina costumam ser mais indiretas e sutis, podem passar despercebidas pelas pessoas em diferentes contextos, inclusive pelas próprias meninas, que muitas vezes não se reconhecem como agressivas.

O desenho transversal utilizado impõe uma limitação à análise dos dados ao não permitir falar em causalidade, embora facilite o aproveitamento dos dados já coletados. Há uma discrepância cronológica entre as variáveis analisadas, pois o uso de substâncias e os comportamentos violentos foram investigados quanto à sua ocorrência no ano anterior às entrevistas, a vitimização por pares foi investigada em sua ocorrência na vida e a relação com pai e mãe, assim como o sofrimento psíquico, refere-se ao momento das entrevistas. No entanto, o delineamento proposto permite tolerar tal discrepância, detendo-se a estudar a possibilidade de associação entre tais eventos.

Outro dado a ser ressaltado em relação ao envolvimento com comportamentos violentos no ano refere-se a uma diferença entre os escolares deste estudo e escolares portugueses. Os adolescentes portugueses de maior idade (15 anos) se envolveram menos em brigas que os de 11 e 13 anos, não havendo diferença entre as faixas etárias em relação ao porte de armas (Matos et al., 2015). Isso sugere que este comportamento diminui com o tempo. Ao contrário, entre os escolares deste estudo há indícios de aumento do envolvimento em comportamentos violentos no ano. Entre os 14 (7,3\%) e os 15 anos de idade $(7,1 \%)$, os escolares referiram maior envolvimento com os dois comportamentos violentos estudados (uso de armas e envolvimento em brigas).

$O$ aumento da agressividade com a idade pode ser interpretado à luz da complexidade dos processos proximais ao longo do ciclo vital (Nasio, 2011). Embora este seja um comportamento negativo e de risco, pode ter a intenção de adaptação às novas demandas psicossociais ou indicar a busca pela integração em um grupo de pares que valida a violência como forma de relação (Berger \& Rodkin, 2011). Este resultado é ainda mais preocupante com a constatação de que é expressivo $(60,3 \%)$ o efeito direto da vitimização por pares sobre o número de comportamentos violentos com os quais os escolares se envolveram no ano. Nota-se que o maior envolvimento dos escolares com comportamentos violentos recebe influência da relação negativa com os pais e do próprio sofrimento psíquico, evidentes na estimativa de efeito indireto (39,7\%). No entanto, a vitimização parece ser suficiente para o envolvimento em comportamentos violentos, contribuindo para a formação de um ciclo de violência. Devido ao delineamento proposto para este estudo, não é possível compreender as causas para este ciclo. No entanto, mesmo supondo que o envolvimento em brigas e o porte de armas tenham sido comportamentos assumidos pelos escolares vitimizados como autodefesa, ambos são comportamentos violentos que tendem a aumentar a ocorrência da violência.

Os resultados deste estudo indicam, ainda, que a vitimização insere os escolares no papel de agressores, ampliando a sua exposição a fatores de risco e comprometendo o seu desenvolvimento. Evidenciam, assim, uma provável naturalização da violência, a qual costuma ocorrer especialmente diante da necessidade de defesa em situações de agressão ou desqualificação (Potocnjak et al., 2011). Com isso, as ações de prevenção ou mesmo de enfrentamento de comportamentos violentos entre jovens devem focar diretamente a prevenção e o enfrentamento das situações de vitimização por pares.

O fato de a relação com os pais ter influenciado menos o efeito da vitimização sobre o envolvimento em comportamentos violentos não significa que pais ou cuidadores não devam ser envolvidos nas intervenções. É possível que, independentemente da relação com os genitores e das suas condições de saúde mental, os escolares não estejam encontrando formas mais adequadas de lidar com as situações de vitimização do que a perpetração da violência como resposta para a violência sofrida. Deve-se considerar que os contextos 
escolares brasileiros estão cirscunscritos pela violência urbana, o que pode influenciar o comportamento dos jovens. Ainda, é possível que o modelo e a pressão de grupos de pares que valorizam a violência sejam mais importantes enquanto influência para a agressividade do que a relação com os pais. Estimular o diálogo e o apoio das famílias pode auxiliar na identificação de situações de vitimização e contribuir para que os jovens busquem formas mais saudáveis de resolver tais situações, ao invés de se envolverem ainda mais em um ciclo de violência.

Tomando-se em conjunto os desfechos aqui estudados, policonsumo de substâncias e número de comportamentos violentos com os quais os escolares se envolveram no ano, nota-se que aumentaram conforme a idade. Do ponto de vista desenvolvimental, o avanço da idade associa-se à necessidade de resolução das tarefas típicas da adolescência, tais como a construção da identidade, o afastamento dos pais, o desenvolvimento da autonomia, a definição profissional e o desenvolvimento do papel sexual (Nasio, 2011). Este conjunto de desafios pode acarretar maior vulnerabilidade emocional dos adolescentes, representada no modelo pelo papel mediador do sofrimento psíquico ao lado da percepção da satisfação com a relação com os pais. Somando-se à pressão dos grupos de pares, à própria curiosidade ou mesmo à necessidade de transgredir (Malta et al., 2014; Vázquez Fernández et al., 2014), o uso de várias substâncias e os comportamentos violentos podem ser opções de enfrentamento de dilemas internos. Tais opções preocupam, pois podem repercutir no desenvolvimento físico, cognitivo, social e afetivo dos adolescentes, com risco de comprometimento, também, do desenvolvimento na vida adulta.

\section{Considerações finais}

Este estudo mostrou que, entre escolares de $12 \mathrm{a}$ 17 anos de duas cidades do sul do Brasil, que sofreram vitimização por pares, uma relação negativa com os pais e a presença de sofrimento psíquico aumentam a possibilidade de uso de substâncias psicoativas e de comportamentos violentos (especificamente, envolvimento em brigas e porte e/ou uso de armas). Assim, a prevenção do envolvimento de jovens nessas situações deve incluir maior atenção às situações de vitimização por pares.

Como a vitimização parece ter efeito direto sobre o número de comportamentos violentos praticados pelos jovens, o combate à violência entre pares pode evitar a formação de círculos de reentrada em situações de violência entre adolescentes, seja como vítimas ou como agressores. Pais e mães de escolares que sofreram vitimização por pares devem ser apoiados, entre outras medidas de promoção de saúde mental durante a adolescência.

Em relação ao policonsumo de substâncias, a qualidade da relação com pais e mães e o menor grau de sofrimento psíquico podem proteger aqueles adolescentes que sofreram vitimização por pares. É possível que outros fenômenos também interfiram na relação entre vitimização e uso de substância. Nesse sentido, um tema importante para futuras investigações é a qualidade das relações de amizade dos adolescentes.

Ressalta-se, ainda, que a tendência das meninas de maior envolvimento com o policonsumo de substâncias e o maior envolvimento dos meninos com comportamentos violentos remete a questões de gênero. Sugere-se que tais aspectos sejam considerados por pais, educadores e profissionais em ações voltadas à saúde e que adolescentes sejam estimulados a participar de discussões sobre gênero, comportamento e saúde.

\section{Referências}

Acock, A. C. (2013). Discovering structural equation modeling using stata (Edição Revisada). Stata Press: Texas, USA: 09/2013. VitalBook file.

Bandeira, C. M., \& Hutz, C. S. (2012). Bullying: prevalência, implicações e diferenças entre os gêneros. Psicologia Escolar e Educacional, 16(1), 35-44. doi: 10.1590/S1413-85572012000100004

Benchaya, M. C., Bisch, N. K., Moreira, T. C., Ferigolo, M., \& Barros, H. M. T. (2011). Non-authoritative parents and impact on drug use: the perception of adolescent children. Jornal de Pediatria, 87(3), 238-244. doi: 10.2223/JPED.2089

Berger, C., Lisboa, C. S. M., Cuadros, O., \& Tezanos-Pinto, P. (2016) Adolescent peer relations and socioemotional development in Latin America: Translating international theory into local research. New Directions for Child and Adolescent Development, 152, 45-58. doi: 10.1002/cad.20161

Berger, C., \& Rodkin, P. C. (2011). Group influences on individual aggression and prosociality: Early adolescentes who change peer affiliations. Social Development, 21(2), 396-413. doi: 10.1111/j.1467-9507.2011.00628.x

Brito, I., Precioso, J. A. G., Correia, C., Albuquerque, C., Samorinha, C., Cunha-Filho, H., \& Becoña, E. (2015). Fatores associados ao consumo de álcool na adolescência, em função do género. Psicologia, Saúde \& Doenças, 16(3), 392-410. doi: 10.15309/15psd1603010

Carlini, E. L. A., Noto, A. R., Sanchez, Z. V. D. M., Carlini, C. M. A., Locatelli, D. P., Abeid, L. R., \& Moura, Y. G. (2010). VI Levantamento Nacional sobre o consumo de drogas psicotrópicas entre estudantes do Ensino Fundamental e Médio das redes pública e privada de ensino nas 27 capitais brasileiras - 2010. Brasília, DF: SENAD. 
Efeitos da vitimização por pares sobre o uso de substâncias psicoativas e comportamentos violentos em adolescentes

Crawford, A. M., \& Manassis, K. (2011). Anxiety, social skills, friendship quality, and peer victimization: An integrated model. Journal of Anxiety, 25, 924-931. doi: 10.1016/j.janxdis.2011.05.005

Cruzeiro, A. L. S., Silva, R. A., Horta, B. L., Souza, L. D. M., Faria, A. D., Pinheiro, R. T., ... Ferreira, C. D. (2008). Prevalência e fatores associados ao transtorno da conduta entre adolescentes: um estudo de base populacional. Cadernos de Saúde Pública, 24(9), 2013-2020. doi: 10.1590/S0102-311X2008000900007

Forlim, B. G., Stelko-Pereira, A. C., \& Williams, L. C. A. (2014). Relação entre bullying e sintomas depressivos em estudantes do ensino fundamental. Estudos de Psicologia, 31(3), 367-375. doi: 10.1590/0103-166x2014000300005

Herrenkohl, T. I., Hemphill, S. A., Mason, W. A., Toumbourou, J. W., \& Catalano, R. F. (2012). Predictors and responses to the growth in physical violence during adolescence: a comparison of students in Washington State and Victoria, Australia. American Journal of Orthopsychiatry, 82(1), 41-49. doi: 10.1111/j.1939-0025.2011.01139.x

Hong, J. S., \& Espelage, D. L. (2012). A review of research on bullying and peer victimization in school: an ecological system analysis. Agression and Violent Behavior, 17, 311-322. doi: 10.1016/j.avb.2012.03.003

Horta, C. L., Horta, R. L., Mester, A., Lindern, D., Weber, J. L. A., Levandowski, D. C., \& Lisboa, C. S. M. (2018). Bullying e uso de substâncias psicoativas na adolescência: uma revisão sistemática. Ciência \& Saúde Coletiva, 23(1), 123-139. doi: 10.1590/1413-81232018231.20932015

Horta, C. L., Lisboa, C. S, M., Teixeira, V. A., Wendt, G. W., \& Horta, R. L. (2015). Violence exposure and substance use among southern Brazilian schoolchildren. Psychology, Society, \& Education, 7(2), 119-132. doi: 10.25115/psye.v7i2.528

Horta, R. L., Horta, B. L., Costa, A. W. N., Prado, R. R., OliveiraCampos, M., \& Malta, D. C. (2014). Uso na vida de substâncias ilícitas e fatores associados entre escolares brasileiros, Pesquisa Nacional de Saúde do Escolar (PeNSE 2012). Revista Brasileira de Epidemiologia, Supl. PeNSE , 17(Suppl. 1), 31-45. doi: 10.1590/1809-4503201400050004

Horta, R. L., Horta, B. L., Pinheiro, R. T., \& Krindges, M. (2010). Comportamentos violentos de adolescentes e coabitação parento-filial. Revista de Saúde Pública [online], 44(6), 979-985. doi: 10.1590/S0034-89102010005000042

Jordão, A. B., \& Ramires, V. R. R. (2010). Adolescência e organização de personalidade borderline: caracterização dos vínculos afetivos. Paidéia, 20(47), 421-430. doi: 10.1590/S0103-863X2010000300014

Kline, R. B. (2005). Methodology in the social sciences. Principles and practice of structural equation modeling ( $2^{\mathrm{a}}$ ed.). Nova lorque, NY: Guilford Press.

Malta, D. C., Oliveira-Campos, M., Prado, R. R., Andrade, S. S. C., Mello, F. C. M., Dias, A. J. R., \& Bomtempo, D. B. (2014). Uso de substâncias psicoativas, contexto familiar e saúde mental em adolescentes brasileiros, Pesquisa Nacional de Saúde dos Escolares (PeNSE 2012). Revista Brasileira de Epidemiologia, 17(Suppl. 1), 46-61. doi: 10.1590/1809-4503201400050005

Matos, M. G., Simões, C., Camacho, I., Reis, M., \& Equipa Aventura Social. (2015). A saúde dos adolescentes portugueses em tempos de recessão - dados nacionais do estudo HBSC de 2014. Lisboa: Centro de Malária e Outras Doenças Tropicais. Recuperado de http://aventurasocial.com/arquivo/1437158618_RELATORIO\%20 HBSC\%202014e.pdf
Mari, J. J., \& Willians, P. A. (1986). A validity study of a psychiatric screening questionnaire (SRQ-20) in primary care in city of São Paulo. The British Journal of Psychiatry, 148, 23-26. doi: 10.1192/bjp.148.1.23

Ministério da Saúde - MS (2010). Secretaria de Atenção em Saúde, Departamento de Ações Programáticas Estratégicas. Diretrizes nacionais para a atenção integral à saúde de adolescentes e jovens na promoção, proteção e recuperação da saúde. Brasília: Autor. Recuperado de http://bvsms.saude.gov.br/bvs/publicacoes/ diretrizes_nacionais_atencao_saude_adolescentes_jovens_promocao_saude.pdf

Nascimento, E. F., Gomes, R., \& Rebello, L. E. F. S. (2009). Violência é coisa de homem? A "naturalização" da violência nas falas de homens jovens. Ciência \& Saúde Coletiva, 14(4), 1151-1157. doi: $10.1590 / S 1413-81232009000400021$

Nasio, J. D. (2011). Como agir com um adolescente difícil? Um livro para pais e profissionais. Rio de Janeiro: Zahar.

Peinado, J., Villanos, M. T., Singh, N., \& Leiner, M. (2014). The association between exposure to violence, alcohol, and drugs and psychosocial and behavioral outcomes among

Mexican-American adolescents of low socioeconomic status. Adiciones, 26(1), 27-33. doi: 10.20882/adicciones.125

Potocnjak, M., Berger, C., \& Tomicic, T. (2011). Uma aproximación relacional a la violência escolar entre pares em adolescentes chilenos: perspectiva adolescente de los factores intervenientes. Psykhe, 20(2), 39-52. doi: 10.4067/S0718-22282011000200004

Romaní, F., \& Gutiérrez, C. (2010). Auto-reporte de victimización escolar y factores asociados en escolares peruanos de educación secundaria, año 2007. Revista Peruana de Epidemiologia, 14(3), 1-9. Recuperado de http://sisbib.unmsm.edu.pe/BVRevistas/epidemiologia/v14_n3/pdf/a06v14n3.pdf

Santos, K. O. B., Araújo, T. M., Pinho, P. S., \& Silva, A. C. C. (2010). Avaliação de um instrumento de mensuração de morbidade psíquica: estudo de validação do Self-Reporting Questionnaire (SRQ-20). Revista Baiana de Saúde Pública, 34(3), 544-560. doi: 10.22278/2318-2660.2010.v34.n3.a54

Souza, D. P. O., \& Silveira Filho, D. X. (2007). Uso recente de álcool, tabaco e outras drogas entre estudantes adolescentes trabalhadores e não trabalhadores. Revista Brasileira de Epidemiologia, 10(2), 276-287. doi: 10.1590/S1415-790X2007000200015

Stata (versão 13.0) (Programa de computador). College Station, USA: StataCorp.

Tavares, B. F., Beria, J. U., \& Lima, M. S. (2001). Prevalência do uso de drogas e desempenho escolar entre adolescentes. Revista de Saúde Pública, 35(2), 150-158. doi: 10.1590/S0034-89102001000200008

Tortorelli, M. F. P., Carreiro, L. R. R., \& Araújo, M. V. (2010). Correlações entre a percepção da violência familiar e o relato de violência na escola entre alunos da cidade de São Paulo. Psicologia Teoria e Prática, 12(1), 32-42. Recuperado de http://pepsic.bvsalud.org/pdf/ ptp/v12n1/v12n1a04.pdf

Vásquez Fernández, M. E., Muñoz Moreno, M. F., Fierro Urturi, A., Alfaro González, M., Rodríguez Carbajo, M. L., \& Rodríguez Molinero, L. (2014). Consumo de sustancias adictivas en los adolescentes de 13 a 18 años y otras conductas de riesgo relacionadas. Pediatría Atención Primaria, 16, 125-134. doi: 10.4321/S1139-76322014000300005

Wang, J., lannotti, R. J., \& Nansel, T. R. (2009). School bullying among adolescents in the United States: physical, verbal, relational, and cyber. Journal of Adolescent Health, 45(4), 368-375. doi: 10.1016/ j.jadohealth.2009.03.021 
Cristina Lessa Horta, Doutora em Psicologia Clínica pela Pontifícia Universidade Católica do Rio Grande do Sul - PUC-RS, é Psicóloga da Pontifícia Universidade Católica do Rio Grande do Sul - PUC-RS.

Endereço para correspondência: Av. Ipiranga, 6681, prédio 11, sala 929, CEP 90619-900 - Porto Alegre/RS.

Telefone: (51) 3320.3500. Email: cristinalessahorta@gmail.com

Rogério Lessa Horta, Doutor em Psicologia pela Pontifícia Universidade Católica do Rio Grande do Sul - PUC-RS, é Professor Adjunto da Universidade FEEVALE. Email: rogeriohortamed@gmail.com

Daniela Centenaro Levandowski, Doutora em Psicologia do Desenvolvimento pela Universidade Federal do Rio Grande do Sul - UFRGS, Pós-Doutora em Psicologia pela Pontifícia

Universidade Católica do Rio Grande do Sul - PUC-RS, é Professora Associada da Universidade Federal de Ciências da Saúde de Porto Alegre - UFCSPA e Bolsista Produtividade em Pesquisa do CNPq. Email: danielal@ufcspa.edu.br

Vanessa Andina Teixeira, Doutora em Saúde Coletiva pela Universidade do Vale do Rio dos Sinos - Unisinos, é Líder Nacional do Núcleo de Apoio Psicopedagógico da rede Laureate International Universities e Professora do Centro Universitário Ritter dos Reis - UniRitter. Email: vanessa.andina@gmail.com

Carolina Saraiva de Macedo Lisboa, Doutora em Psicologia do Desenvolvimento pela Universidade Federal do Rio Grande do Sul - UFRGS com estágio na Universidade do Minho em

Portugal, é Professora da Pontifícia Universidade Católica do Rio Grande do Sul - PUC-RS. Email: carolina.lisboa@pucrs.br

Recebido em 28.dez.17 Revisado em 24.jul.19 Aceito em 31.dez.19 